03

\title{
Влияние жидкой фазы на параметры и пределы пузырьковой детонации
}

\author{
(C) А.В. Пинаев, Е.С. Прохоров
}

Институт гидродинамики им. М.А. Лаврентьева СО РАН, 630090 Новосибирск, Россия

e-mail: avpin@ngs.ru

(Поступило в Редакцию 1 марта 2017 г.)

Экспериментально исследован процесс сжатия и воспламенения пузырьков с реагирующим газом в волнах пузырьковой детонации, установлено существенное влияние вязкости жидкости на параметры детонации. Численно показано, что учет зависимости показателя адиабаты от температуры на стадии сжатия пузырька приводит к значительному уменьшению конечных значений давления и температуры. В рамках равновесной модели рассчитаны параметры продуктов сгорания реагирующего газа в пузырьке и исследовано влияние на эти параметры жидкости, поступающей внутрь пузырька в виде микрокапель и паровой фазы.

DOI: 10.21883/JTF.2017.12.45221.2225

Пузырьковая детонация (ПД) впервые экспериментально обнаружена и исследована А.В. Пинаевым и А.И. Сычевым в средах с малым энерговыделением химически инертной жидкости с пузырьками взрывчатого газа $[1,2]$ и горючей жидкости с пузырьками кислорода [3-5]. Известно, что с ростом вязкости жидкости $\mu_{f}$ при сжатии газового пузырька, согласно теории Рэлея, увеличиваются потери энергии и уменьшаются конечные давление и температура газа. Однако опыты в той же постановке дают прямо противоположные результаты. Этот экспериментальный результат имеет важное прикладное значение для химически активных и инертных пузырьковых сред, и возникает необходимость в постановке и решении задачи о влиянии жидкой фазы в объеме пузырька на параметры сжимаемого газа. Несмотря на интерес к „пузырьковой тематике“, опубликовано небольшое количество работ, посвященных исследованию сильных (10-100 МРа) ударных и детонационных волн в пузырьковых средах. Цель настоящей работы - частично восполнить этот пробел, экспериментально изучить сжатие пузырька в волне пузырьковой детонации и параметры ПД в широком диапазоне изменения $\mu_{f}$, рассчитать параметры газа в зависимости от количества жидкой фазы в объеме пузырька и ее предельную концентрацию, при которой газ в пузырьке не воспламеняется.

Опыты проведены в вертикальной ударной трубе диаметром $35 \mathrm{~mm}$, длиной $5 \mathrm{~m}$ при стандартных начальных условиях. Генератор пузырьков создавал пузырьки размером $d_{0}=2.5-4 \mathrm{~mm}$ при объемном газосодержании в системе $\beta_{0}=0.5-6 \%$. Относительная ошибка определения $\beta_{0}$, давления и скорости $D$ пузырьковой детонации не превышала 5\%. Исследованы водноглицериновые растворы с объемным содержанием глицерина $0 \leq G_{f} \leq 70 \%$, вязкость жидкости изменялась в пределах $10^{-3} \leq \mu_{f} \leq 27.1 \cdot 10^{-3}$ Pa s.

Из фоторазверток свечения волн пузырьковой детонации и профилей давления, свечения и кривых из- менения безразмерного диаметра пузырьков $n=d / d_{0}$ (рис. 1) установлено, что $n_{\min }$ расположено вблизи начала воспламенения, величина $1 / n_{\min } \approx 3-4$, время от начала взаимодействия пузырька с волной ПД до воспламенения составляет 20-30 $\mu \mathrm{s}$. Влияние свойств жидкого компонента проиллюстрировано на примере систем с различным содержанием глицерина (рис. 2) с увеличением $G_{f}$ и $\mu_{f}$ возрастает скорость пузырьковой детонации, наиболее заметное влияние вязкости

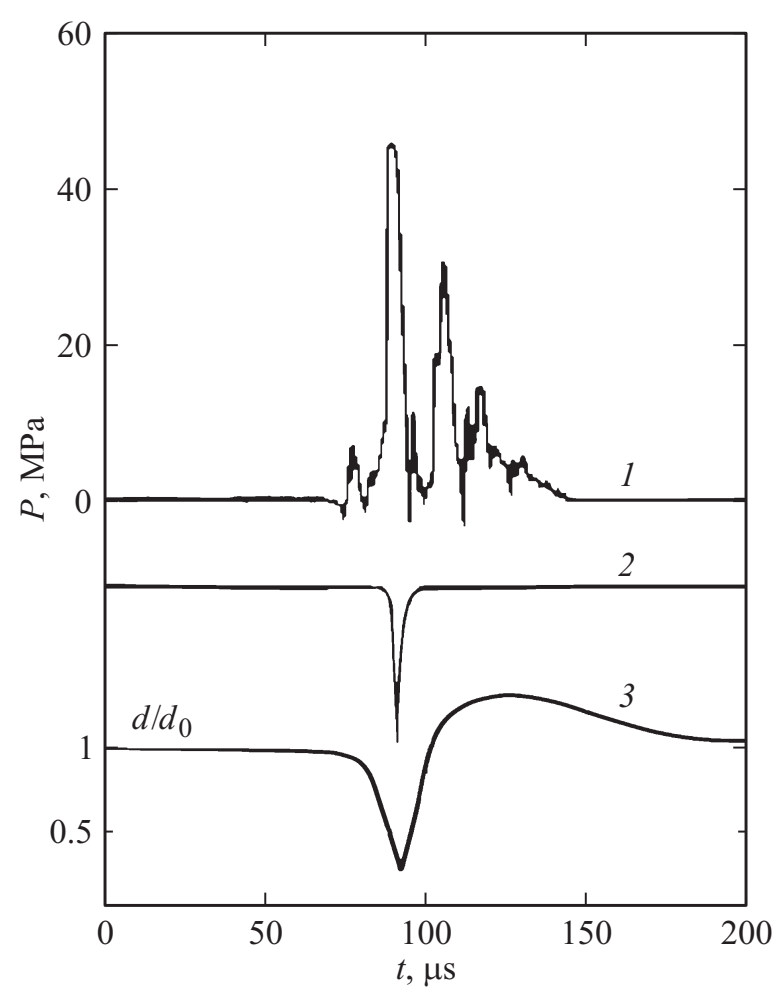

Рис. 1. Осциллограммы профилей давления (1), свечения (2) и кривые сжатия пузырьков (3) в волне пузырьковой детонации; $\beta_{0}=2 \%,\left(0.5 \mathrm{H}_{2} \mathrm{O}+0.5\right.$ глицерин $)-\left(\mathrm{C}_{2} \mathrm{H}_{2}+2.5 \mathrm{O}_{2}\right)$. 


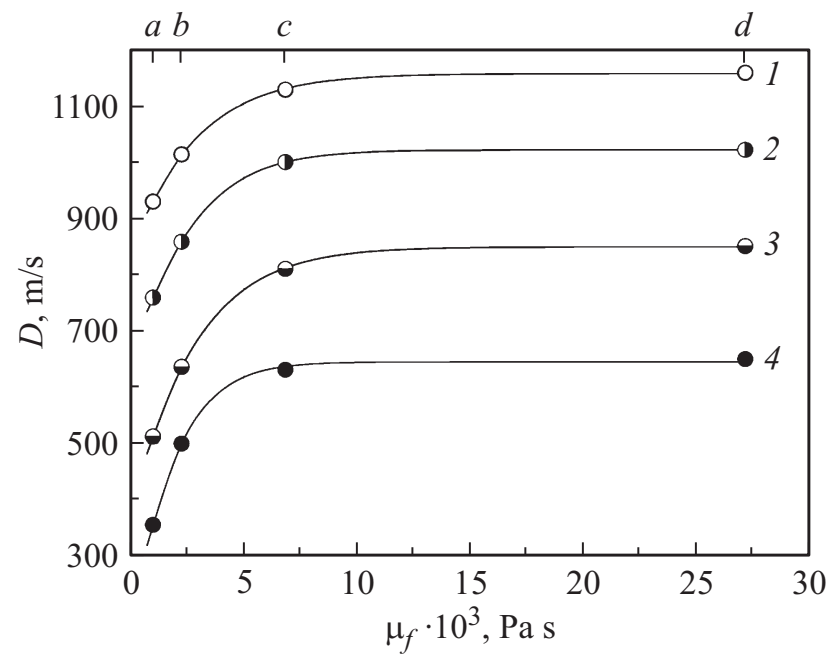

Рис. 2. Влияние вязкости жидкости на установившуюся скорость пузырьковой детонации; $\left(\mathrm{C}_{2} \mathrm{H}_{2}+2.5 \mathrm{O}_{2}\right) ; \beta_{0}, \%=1(1)$, 2 (2), 4 (3), 6 (4); $a-d-$ водно-глицериновые жидкости: $G_{f}=0,0.25,0.5,0.7, \mu_{f} \cdot 10^{3}, \mathrm{~Pa} \mathrm{~s}=1.01,2.27,6.84,27.10$.

на величину $D$ наблюдается при $\mu_{f} \leq 10^{-2} \mathrm{Pas}$, если $\mu_{f}<10^{-3} \mathrm{Pas}$, то ПД в газожидкостной среде не распространяется.

На покадровых фотографиях видно, что с увеличением вязкости жидкости начальная форма пузырьков становится ближе к сферической, поверхность гладкой. Это способствует уменьшению теплопотерь и увеличению интенсивности ПД. В маловязких жидкостях свободно всплывающие пузырьки имеют эллипсоидальную форму, при их сжатии внутри пузырька образуется кумулятивная струйка жидкости. Искажение формы пузырьков наблюдается уже при первом расширении, после $2-3$ колебаний пузырьки полностью утрачивают первоначальную форму и распадаются на мелкие части. Во всех опытах с ростом $\mu_{f}$ усиливается роль вязкостной стабилизации поверхности пузырьков и как следствие, уменьшаются поступление жидкой фазы внутрь пузырьков и тепловые потери. Причем такое „положительное“ влияние вязкости намного превышает вязкостные диссипативные потери в уравнении Рэлея [6].

Численно исследована динамика роста давления $p$ и температуры $T$ смеси $\mathrm{C}_{2} \mathrm{H}_{2}+2.5 \mathrm{O}_{2}$ внутри отдельного пузырька при его адиабатическом сжатии. Расчеты выполнены с постоянным показателем адиабаты $\gamma_{0}\left(T_{0}\right)$ и с переменным $\gamma(T)$. Для аппроксимации термодинамических функций газовой смеси использованы табличные данные [7]. Установлено, что учет зависимости $\gamma=\gamma(T)$ приводит к существенному уменьшению значений $p$ и $T$ при сжатии по сравнению с расчетами с постоянным $\gamma_{0}$. Например, при уменьшении диаметра пузырька в 4 раза давление и температура газа в нем на $23 \%$ меньше, чем в расчетах с постоянным $\gamma_{0}$. Влияние непостоянства $\gamma(T)$ ранее не учитывалось при теоретическом анализе ПД в работах других авторов. На рис. 3 сплошные ли- нии - расчет изменения $p, T$ и периода индукции $\tau_{i}$ для смеси $\mathrm{C}_{2} \mathrm{H}_{2}+2.5 \mathrm{O}_{2}$ при сжатии пузырька. Для расчета $\tau_{i}$ использована кинетическая зависимость [8]: $\lg \left(\tau\left[\mathrm{O}_{2}\right]^{1 / 2}\left[\mathrm{C}_{2} \mathrm{H}_{2}\right]^{1 / 2}\right)=10.81+E_{a} /(4.58 T)$, где энергия активации $E_{a}=17.3 \mathrm{kcal} / \mathrm{mol}, \tau_{i}$ имеет размерность $s$, а концентрации молекул кислорода $\left[\mathrm{O}_{2}\right]$ и ацетилена $\left[\mathrm{C}_{2} \mathrm{H}_{2}\right]$ в смеси - mol/l.

Поскольку при сжатии пузырька параметры $p$ и $T$ меняются, то время задержки воспламенения $t_{*}$ химически реагирующей смеси определяется общепринятым условием:

$$
\int_{0}^{t_{*}} \frac{d t}{\tau_{i}}=1 .
$$

На основании выполненных экспериментов можно принять $t_{*} \approx 20-30 \mu \mathrm{s}$. Полагая, что диаметр пузырька в течение $0 \leq t \leq t_{*}$ линейно зависит от времени $t$, с помощью (1) можно оценить, при какой степени сжатия будет происходить взрыв газа внутри пузырька. Расчеты показывают, что при $t_{*}=30 \mu \mathrm{s}$ взрыв газа происходит при уменьшении $d_{0}$ в 3.55 раза, а при $t_{*}=20 \mu \mathrm{s}-$ в 3.7 раза. Эти предельные величины для $d_{0} / d$ хорошо согласуются с экспериментальными данными. При этом, чем меньше $t_{*}$, тем более высокие значения $p$ и $T$ должен иметь реагирующий газ перед взрывом (рис. 3).

При расчете параметров сгорания газовой смеси в замкнутом объеме использована модель для анализа равновесных течений химически реагирующих газов [9], которая примерно на порядок точнее модели [10], используемой в [11] для расчета пузырьковой детонации. Такого повышения точности удалось добиться за счет использования более адекватных уравнений для расчета изменения молярной массы $\mu=\mu(p, T)$ и удельной внутренней энергии $U=U_{\text {th }}+U_{\text {ch }}=U(\mu T)$, в том числе ее термодинамической $U_{\text {th }}$ и химической $U_{\mathrm{ch}}$ частей, при сдвиге химического равновесия продуктов детонационного сгорания углеводородов. При этом химический состав ПД характеризуется только относительными концентрациями атомов кислорода $n_{\mathrm{O}}$, углерода $n_{\mathrm{C}} \quad\left(n_{\mathrm{C}} \leq n_{\mathrm{O}}\right)$, водорода $n_{\mathrm{H}}$, азота $n_{\mathrm{N}}$ и любых других одноатомных инертных веществ - $n_{Z}$, а продукты химической реакции могут иметь только следующий равновесный состав (доля остальных веществ незначительна):

$$
\begin{aligned}
v_{\mathrm{CO}_{2}} \mathrm{CO}_{2} & +v_{\mathrm{H}_{2} \mathrm{O}} \mathrm{H}_{2} \mathrm{O}+v_{\mathrm{CO}} \mathrm{CO}+v_{\mathrm{OH}} \mathrm{OH}+v_{\mathrm{O}_{2}} \mathrm{O}_{2} \\
& +v_{\mathrm{H}_{2}} \mathrm{H}_{2}+v_{\mathrm{N}_{2}} \mathrm{~N}_{2}+v_{\mathrm{O}} \mathrm{O}+v_{\mathrm{H}} \mathrm{H}+v_{\mathrm{Z}} \mathrm{Z}
\end{aligned}
$$

где $v_{s}$ - мольная доля вещества $s\left(\sum_{s} v_{s}=1, \mu=\sum_{s} v_{s} \mu_{s}\right)$. Дополнительный анализ показывает, что такой подход весьма перспективен при моделировании горения и детонации в средах с переменным химическим составом. Поэтому отпадает необходимость при вычислительном итерационном процессе каждый раз дополнительно ре- 

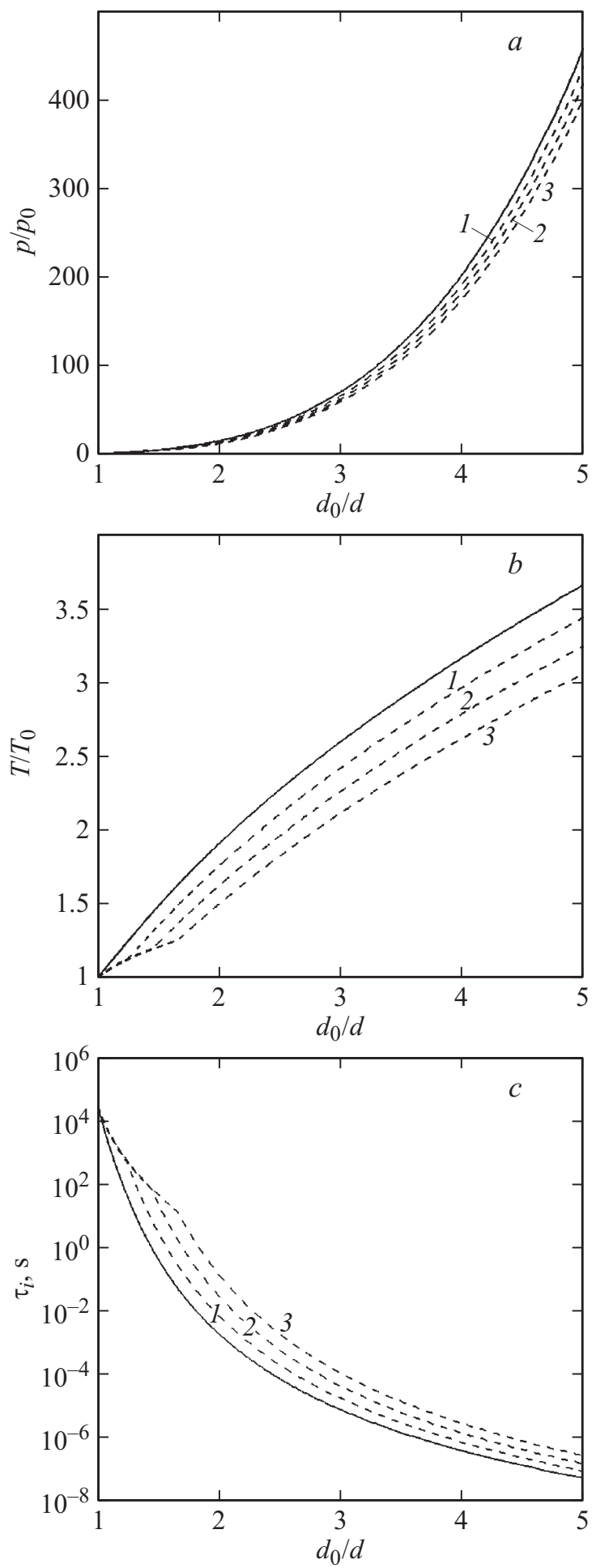

Рис. 3. Зависимости давления $(a)$, температуры $(b)$ и периода индукции $(c)$ внутри пузырька при его сжатии. Сплошные линии - расчеты без микрокапель воды. Штриховые линии расчеты с учетом наличия микрокапель воды, отношение массы воды к массе газа $m_{2} / m_{1}=0.01(1), 0.02(2), 0.03(3)$.

шать (как, например, при точной постановке [12]) расширенную систему уравнений, включающую формулы химического равновесия, термохимические соотношения и формулы атомарного баланса для всех возможных компонентов продуктов реакции.

В результате получим расчетные параметры продуктов сгорания смеси $\mathrm{C}_{2} \mathrm{H}_{2}+2.5 \mathrm{O}_{2}$ после взрыва в замкнутом объеме для интервала $d_{0} / d=3.55-3.7$ : плотность $\rho=55.4-63.2 \mathrm{~kg} / \mathrm{m}^{3}, \quad p=93.9-108.1 \mathrm{MPa}$, $T=5132-5183 \mathrm{~K}$. Отметим, что отличие этих значений от точного решения [12] меньше $1 \%$.

Для исследования влияния на период индукции $\tau_{i}$ и задержку воспламенения $t_{*}$ массы воды $m_{2}=m_{2 l}+m_{2 g}$, которая может присутствовать внутри пузырька в виде микрокапель (с массой $m_{2 l}$ ) и паровой фазы (с массой $m_{2 g}$ ) была усложнена постановка задачи путем введения дополнительных членов в уравнение энергетического баланса термодинамической системы при адиабатическом сжатии. В расчетах варьировалось отношение $m_{2} / m_{1}$, где $m_{1}-$ масса реагирующего газа, заключенного в пузырьке. Полагали, что перед сжатием пузырька некоторая масса воды в его объеме сосредоточена в жидкой фазе. По мере увеличения температуры вода начинает испаряться. Вычислительный алгоритм построен таким образом, что парциальное давление паров воды не превышало давления насыщенного пара для текущей температуры внутри пузырька. Соответствующие результаты расчетов представлены на рис. 3 в виде штриховых линий. Видно, что потери энергии за счет нагрева и испарения воды, приводят к снижению значений $p$ и $T$ при сжатии пузырька, при этом период индукции $\tau_{i}$ возрастает. Точки перегиба на кривых соответствуют моменту полного испарения воды внутри пузырька.

Анализ расчетов показал, что параметр $m_{2} / m_{1}$ существенно влияет на задержку воспламенения $t_{*}$. Например, если $m_{2} / m_{1}>0.015$, то $t_{*}>30 \mu \mathrm{s}$ даже при уменьшении диаметра в 4 раза. Поэтому в рамках рассмотренной модели $m_{2} / m_{1}=1.5 \%$ можно считать критической концентрацией жидкости, приводящей к срыву воспламенения пузырьков и пузырьковой детонации.

Таким образом, для отдельного пузырька в волне ПД, экспериментально получена зависимость от времени степени сжатия и определен промежуток времени от начала сжатия пузырька до его воспламенения. Установлено, что с ростом $\mu_{f}$ усиливается роль вязкостной стабилизации поверхности пузырьков, и, как следствие, уменьшаются поступление жидкой фазы внутрь пузырьков и тепловые потери. Показано, что учет зависимости показателя адиабаты газа от температуры на стадии сжатия пузырька оказывает существенное влияние на давление и температуру газовой смеси в пузырьке перед его воспламенением. В зависимости от содержания воды внутри пузырька рассчитаны параметры продуктов сгорания реагирующей газовой смеси в замкнутом объеме и определена критическая концентрация жидкости, приводящая к срыву пузырьковой детонации.

Работа выполнена при частичной финансовой поддержке РФФИ (№ 15-01-01154a). 


\section{Список литературы}

[1] Сычев А.И., Пинаев А.В. // Тез. I Всесоюз. симп. по макроскопической кинетике и химической газодинамике. Алма-Ата, Черноголовка, 1984. Т. 1, Ч. 1. № 65. С. 54-55.

[2] Сычев А.И., Пинаев А.В. // ПМТФ. 1986. № 1. С.133-138.

[3] Пинаев А.В., Сычев А.И. // ДАН СССР. 1986. Т. 290. № 3. C. 611-615.

[4] Пинаев А.В., Сычев А.И. // ФГВ. 1986. Т. 22.№ 3. C. $109-118$.

[5] Пинаев А.В., Сычев А.И. // ФГВ. 1987. Т. 23. № 6. С. 76-84.

[6] Pinaev A.V., Prokhorov E.S. // Tр. Всерос. школы-конф. с междунар. участием „Химия и физика горения и дисперсных систем“, посвященной 110-летию со дня рождения члена-корреспондента АН СССР А.А. Ковальского. Новосибирск, ИХКГ СО РАН, 2016 г. С. 1-5.

[7] Термодинамические свойства индивидуальных веществ / Под ред. В.П. Глушко. М.: Наука, 1978. Т. 1, кн. 1. 495 с.; кн. $2.327 \mathrm{c}$.

[8] White D.R. // Proc. XI Intern. Symp. on Combustion. Pittsburg: Acad. Press, 1967. P. 147-154.

[9] Прохоров Е.С. // ФГВ. 1996. Т. 32. № 3. С. 77-85.

[10] Николаев Ю.А., Зак Д.В. // ФГВ. 1988. Т. 24. № 4. С. 87-90.

[11] Троцюк А.В., Фомин П.А. // ФГВ. 1992. Т. 28. № 4. C. $129-136$.

[12] Николаев Ю.А., Топчиян М.Е. // ФГВ. 1977. Т. 13. № 3. C. $393-404$. 